This is a self-archived - parallel published version of this article in the publication archive of the University of Vaasa. It might differ from the original.

\title{
Self-scheduling approach to coordinating wind power producers with energy storage and demand response
}

Author(s): Jamali, Ali; Aghaei, Jamshid; Esmaili, Masoud; Niknam, Taher; Nikoobakht, Ahmad; Shafie-khah, Miadreza; Catalão, João P. S.

Title: $\quad$ Self-scheduling approach to coordinating wind power producers with energy storage and demand response

Year: $\quad 2019$

Version: Accepted manuscript

Copyright (C)2019 IEEE. Personal use of this material is permitted. Permission from IEEE must be obtained for all other uses, in any current or future media, including reprinting/republishing this material for advertising or promotional purposes, creating new collective works, for resale or redistribution to servers or lists, or reuse of any copyrighted component of this work in other works.

\section{Please cite the original version:}

Jamali, A., Aghaei, J., Esmaili, M., Niknam, T., Nikoobakht, A., Shafie-khah, M., \& Catalão, J.P.S., (2019). Self-scheduling approach to coordinating wind power producers with energy storage and demand response. IEEE transactions on sustainable energy. http://doi.org/10.1109/TSTE.2019.2920884 


\title{
Self-Scheduling Approach to Coordinating Wind Power Producers with Energy Storage and Demand Response
}

\author{
Ali Jamali, Jamshid Aghaei, Senior Member, IEEE, Masoud Esmaili, Senior Member, IEEE, \\ Ahmad Nikoobakht, Taher Niknam, Member, IEEE, Miadreza Shafie-khah, Senior Member, IEEE, \\ and João P. S. Catalão, Senior Member, IEEE
}

\begin{abstract}
The uncertainty of wind energy makes wind power producers (WPPs) incur profit loss due to balancing costs in electricity markets, a phenomenon that restricts their participation in markets. This paper proposes a stochastic bidding strategy based on virtual power plants (VPPs) to increase the profit of WPPs in short-term electricity markets in coordination with energy storage systems (ESSs) and demand response (DR). To implement the stochastic solution strategy, the Kantorovich method is used for scenario generation and reduction. The optimization problem is formulated as a MixedInteger Linear Programming (MILP) problem. From testing the proposed method for a Spanish WPP, it is inferred that the proposed method enhances the profit of the VPP compared to previous models.
\end{abstract}

Index Terms-Wind Energy; Energy Storage System; Demand Response; Uncertainty; Stochastic Programming; Electricity Market.

\section{NOMENCLATURE}

\section{Indices}

$t\left(N_{T}\right) \quad$ Timeslot index (number of timeslots).

$\omega\left(N_{\omega}\right) \quad$ Scenario index (number of scenarios).

$l\left(N_{l}\right) \quad$ Linearized segment index (number of segments).

Parameters

$W^{\max } \quad$ Wind unit capacity (MW).

$\lambda^{D}, \lambda^{I}$ Energy price in the DA and intraday markets, respectively $(\$ / \mathrm{MWh})$.

$\rho_{\omega} \quad$ Probability of scenario $\omega$.

$R^{+}, R^{-}$Ratio of positive and negative, respectively, energy

imbalance of WPP with respect to DA market.

$\gamma \quad$ The ratio of WPP offer in the intraday market with respect to the DA market.

$\lambda^{*} \quad$ Payment rate for incentive-based DR (\$/MWh).

J.P.S. Catalão acknowledges the support by FEDER funds through COMPETE 2020 and by Portuguese funds through FCT, under POCI-010145-FEDER-029803 (02/SAICT/2017) and POCI-01-0145-FEDER-006961 (UID/EEA/50014/2019). (Corresponding authors: Miadreza Shafie-khah and João P. S. Catalão).

A. Jamali, J. Aghaei and T. Niknam are with the Department of Electrical and Electronics Engineering, Shiraz University of Technology, Shiraz, Iran (emails: alijamali1367@gmail.com, aghaei@sutech.ac.ir, niknam@sutech.ac.ir).

M. Esmaili is with the Department of Electrical and Electronics Engineering, Islamic Azad University, West Teran, Tehran, Iran (e-mail: msdesmaili@ieee.org).

A. Nikoobakht is with Higher Education Center of Eghlid, Eghlid, Iran(email: a.nikoobakht@eghlid.ac.ir)

M. Shafie-khah is with the School of Technology and Innovations, University of Vaasa, 65200 Vaasa, Finland (e-mail: miadreza@gmail.com)

J.P.S. Catalão is with the Faculty of Engineering of the University of Porto and INESC TEC, Porto 4200-465, Portugal (e-mail: catalao@fe.up.pt). $\sigma \quad$ The coefficient of relationship between energy price and load.

$D_{0} \quad$ The normal value of aggregated loads (MW).

$\eta_{1} \quad$ Upper limit of curtailable load as a fraction of initial load in demand response.

$\mu \quad$ The portion of total interruptible load energy with respect to total initial load energy.

$S \quad$ The slope of linearized segments.

$P^{\max } \quad$ Maximum charging or discharging power of ESS (MW).

$E_{0} \quad$ Initial energy of ESS (MWh).

$d u \quad$ Duration of time periods (h).

$\eta_{c h}, \eta_{d c h}$ Charging and discharging efficiency of ESS.

$E^{\min } \quad$ Minimum energy level of ESS (MWh).

$E^{\max } \quad$ Rated energy of ESS (MWh).

Variables

$P^{D}, P^{I}$ WPP offer in the DA and intraday markets, respectively (MW).

$P^{\text {sch }} \quad$ Scheduled power of WPP (MW).

$W \quad$ Power output of WPP (MW).

$\delta \quad$ Power deviation of WPP from its scheduled value (MW).

$\delta^{+}, \delta^{-}$Positive and negative, respectively, power deviation of WPP with respect to scheduled value (MW).

$L^{D}, L^{I} \quad$ Curtailable load offer of DR in DA and intraday markets, respectively (MW).

$L^{\text {sch }} \quad$ Scheduled curtailable load of DR (MW).

$L^{\text {sch_l }} \quad$ Linearized segments of $L^{s c h}(\mathrm{MW})$.

$P^{c h, D} \quad$ Charging offer of ESS in the DA market (MW).

$P^{d c h, D} \quad$ Discharging offer of ESS in the DA market (MW).

$P^{c h, I} \quad$ Charging offer of ESS in the intraday market (MW).

$P^{d c h, I} \quad$ Discharging offer of ESS in the intraday market (MW).

$y \quad$ Binary variable equal to 1 if the ESS is being charged.

$P^{\text {ch,sch }} \quad$ Scheduled charging offer of ESS (MW).

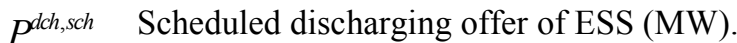

$E^{D} \quad$ Energy of ESS in the DA market (MWh).

$E^{s c h} \quad$ Scheduled energy of ESS (MWh).

$P^{D, V P P} \quad$ Offer of VPP in the DA market (MW).

$P^{I, V P P} \quad$ Offer of VPP in the intraday market (MW). 
$\delta^{+, V P P} \quad$ Positive power deviation of VPP from its scheduled value (MW).

$\delta^{-, V P P} \quad$ Negative power deviation of VPP from its scheduled value (MW).

$L^{\text {sch,VPP }} \quad$ Scheduled curtailable load of VPP (MW).

$L^{\text {sch_l,VPP }}$ Linearized segments of $L^{s c h, V P P}$ for VPP (MW).

$P^{\text {sch,VPP }}$ Scheduled offer of VPP (MW).

$P^{c h, D, V P P}$ Charging offer of VPP in the DA market (MW).

$P^{d c h, D, V P P}$ Discharging offer of VPP in the DA market (MW).

$P^{c h, s c h, V P P}$ Scheduled charging offer of VPP (MW).

$P^{d c h, s c h, V P P}$ Scheduled discharging offer of VPP (MW).

$L^{D, V P P} \quad$ Curtailable load offer of VPP in DA market (MW).

$L^{I, V P P} \quad$ Curtailable load offer of VPP in intraday market (MW).

$E^{D, V P P} \quad$ ESS energy of VPP in the DA market (MWh).

$E^{\text {sch, } V P P}$ Scheduled EES energy of VPP (MWh).

\section{INTRODUCTION}

\section{A. Motivation and Aim}

$\mathrm{R}$ ENEWABLE energy sources such as wind power can be a viable solution to remedy pollutions and greenhouse gases produced by central large power plants. However, uncertainty of wind generation restricts participation of Wind Power Producers (WPPs) in electricity markets due to energy imbalance costs [1].

Although, there are some supportive solutions for WPPs such as assigning subsides or special tariffs in order to keep them in markets, these solutions are less compatible with competitive electricity market principles and therefore, a market-based solution is more preferred to increase the penetration level of renewables [2]. Intraday markets have been introduced to give a chance for WPPs to adjust their bids/offers after gate closure of the Day-ahead (DA) market in order to reduce their imbalance costs. Corrections after DA gate closure not only can be beneficial to increase WPP penetration in electricity markets but also can reduce the energy volume and price of real-time balancing markets [3].

In order to cope with the uncertainty of wind energy and therefore to increase the profit and penetration level of WPPs in electricity markets, Demand Response (DR) and Energy Storage System (ESS) are also used in the literature. From WPP uncertainty point of view, DR provides a flexible load profile to be more consistent with uncertain wind power and finally to reduce WPP uncertainty costs. ESSs can also be used to mitigate energy imbalances in the real-time markets [4]. Accordingly, to cope with the wind power uncertainty, Virtual Power Plants (VPPs) can play an active role in electricity markets. Although, DR, ESS, and VPP are employed in the literature for wind energy applications, there is a research gap to model a VPP that jointly employs DR and ESS to increase the profit of WPP in the DA and intraday markets. This model of VPP makes a higher profit for WPP than existing models and consequently, it better prepares the ground for participation of renewable energy sources in the competitive electricity markets.

\section{B. Literature Review}

Valuable research is available in literature to incorporate WPPs in different electricity markets. For instance, in [5], a bilevel stochastic model is proposed for strategic offering of a WPP with market power in the DA market as a price maker and in the balancing market as a deviator. Authors in [2] proposed a multi-stage risk-constrained model to derive optimal offering strategy of a WPP to participate in DA and balancing markets as a price maker entity. The application of DR and ESS is also addressed in literature.

Authors in [3] suggested a stochastic framework for WPP participation in different electricity markets (DA and balancing markets) considering DR as uncoordinated operation problem. In [6], a strategic bidding is proposed for a WPP using an energy storage facility to participate in DA and real-time markets with modeling the WPP as a price-taker in the markets. In addition, a VPP, which is composed of WPP and DR, is proposed to mitigate wind uncertainty. Authors in [7] formulated the coordinated operation of WPP and a storage unit in DA and hour-ahead markets. Optimal energy and reserve bids are derived and the stochastic problem is converted to a convex optimization to assure the profitability of private investments on storage units. In [8], the optimal bidding, scheduling, and deployment of battery ESS are studied in the California DA energy market by decomposing the stochastic problem into inner and outer subproblems. Authors in [9] studied DR trading in DA markets using a twostep sequential market clearing.

Similarly, the WPP in the DA market model has been proposed by [10]. In [11], a mechanism of intraday market with considering real-time information of WPPs and shiftable loads has been presented. In this reference, the WPPs make decisions to multiple market transactions in different hours based on the market price. Also, the authors of [12] have formulated the model of the energy bidding problem for VPP with its participants in the regular electricity market and the intraday demand response exchange market. Moreover, the coupon-based DR program is used in [13] to coordinate with WPP to obtain optimal operation in the electricity market. In [14] and [15], the DA market model based on DR capability for congestion management with WPP uncertainty has been proposed. Finally, the capability of flexible resources such as DR and ESS to reduce the curtailed wind energy and virtual biding as well as increasing system flexibility have been presented in [16] - [18].

\section{Contributions}

Considering the reviewed literature, the contribution of the current paper is to propose an offering strategy to maximize the profit of a VPP consisting of a WPP, DR, and ESS, entitled wind-demand response-storage, in a coordinated operation in the DA, intraday, and balancing markets. Although these parts of the VPP are separately addressed in the literature, they are not modeled in a coordinated operation by an integrated model of the VPP.

In order to model the uncertainties of wind power and market prices (in DA, intraday, and balancing markets), a scenario-based stochastic programming is used. 
The optimization problem is linearized to enhance its computational efficiency and it is formulated as a MixedInteger Linear Programming (MILP) problem. The Kantorovich method with a fast forward algorithm is employed for scenario generation and reduction. Different case studies with/without DR and ESS are thoroughly studied and compared; it is inferred that the coordinated operation results in a higher profit of the VPP in the three electricity markets compared with existing literature works. To summarize the unique features of the proposed framework with respect to the previous works in the area, the taxonomy of recent works can be seen in Table I.

Noted that the ES system has been considered in our proposed model. Accordingly, the ES system is capable of storing wind energy during the periods when wind price is low to be used during the periods when wind price is high, thus, WPP can be scheduled with the ES system. A proficient way to dispatch the WPP in electricity markets and manage the WPP volatility is to exploit utility-scale energy storage systems. Also, DRs similar to ES system can play an important role in addressing the issue of wind power scheduling.

\section{Paper Organization}

The remaining parts of this paper are organized as follows: In Section II, the problem formulation is described in states with/without DR and ESS and the stochastic method used in the paper is briefly explained. Section III includes numerical results and discussions, and Section IV concludes the paper.

\section{PROBLEM FORMULATION}

In this section, the uncoordinated model of WPP, DR, and ESS is separately represented and afterward, the proposed coordinated model of VPP is presented. In the numerical result section, we follow these models to compare and see the effect of coordinated operation of VPP on its cost.

TABLE I. TAXONOMY OF RECENT WORKS

\begin{tabular}{c|c|c|c|c|c|c|c|c}
\hline \multirow{2}{*}{ Ref } & \multicolumn{3}{|c|}{ Resource } & \multicolumn{4}{c|}{ Market } & \multicolumn{2}{c}{$\begin{array}{c}\text { Coordinated } \\
\text { operation }\end{array}$} \\
\hline & WPP & DR & ESS & DA & Intraday & Balancing & Yes & No \\
\hline$[2]$ & $\times$ & & & $\times$ & & $\times$ & & $\times$ \\
\hline$[3]$ & $\times$ & $\times$ & & $\times$ & & $\times$ & & $\times$ \\
\hline$[5]$ & $\times$ & & & $\times$ & & $\times$ & & $\times$ \\
\hline$[6]$ & $\times$ & & $\times$ & $\times$ & & & & $\times$ \\
\hline$[7]$ & $\times$ & & $\times$ & $\times$ & & & $\times$ & \\
\hline$[8]$ & & & $\times$ & $\times$ & & & & $\times$ \\
\hline$[9]$ & & $\times$ & & $\times$ & & & & $\times$ \\
\hline$[10]$ & $\times$ & & & $\times$ & & & & $\times$ \\
\hline$[11]$ & $\times$ & & & & $\times$ & & & $\times$ \\
\hline$[12]$ & $\times$ & $\times$ & & & $\times$ & & & $\times$ \\
\hline$[13]$ & $\times$ & $\times$ & & $\times$ & & & & $\times$ \\
\hline$[14]$ & $\times$ & $\times$ & & $\times$ & & $\times$ & & $\times$ \\
\hline$[15]$ & $\times$ & $\times$ & & $\times$ & & $\times$ & & $\times$ \\
\hline$[16]$ & $\times$ & $\times$ & $\times$ & $\times$ & & & & $\times$ \\
\hline$[17]$ & $\times$ & $\times$ & & $\times$ & & & $\times$ & \\
\hline$[18]$ & $\times$ & $\times$ & & $\times$ & & & $\times$ & \\
\hline This paper & $\times$ & $\times$ & $\times$ & $\times$ & $\times$ & $\times$ & $\times$ & \\
\hline
\end{tabular}

\section{A. The Uncoordinated Model of WPP}

The proposed basic model for WPP is formulated [3] as:

$$
\begin{gathered}
\operatorname{Max} \\
P_{t, \omega}^{D}, P_{t, \omega}^{I}, \delta_{t, \omega}^{+}, \delta_{t, \omega}^{-}
\end{gathered} \sum_{t=1}^{N_{T}} \sum_{\omega=1}^{N_{\omega}} \rho_{\omega}\left\{\begin{array}{l}
\lambda_{t, \omega}^{D} P_{t, \omega}^{D}+\lambda_{t, \omega}^{I} P_{t, \omega}^{I} \\
+\lambda_{t, \omega}^{D} R_{t, \omega}^{+} \delta_{t, \omega}^{+}-\lambda_{t, \omega}^{D} R_{t, \omega}^{-} \delta_{t, \omega}^{-}
\end{array}\right\}
$$

subject to:

$$
\begin{gathered}
0 \leq P_{t, \omega}^{D} \leq W^{\max } \quad \forall t, \forall \omega \\
P_{t, \omega}^{s c h}=P_{t, \omega}^{D}+P_{t, \omega}^{I} \quad \forall t, \forall \omega \\
0 \leq P_{t, \omega}^{s c h} \leq W^{\max } \quad \forall t, \forall \omega \\
\delta_{t, \omega}=W_{t, \omega}-P_{t, \omega}^{s c h} \quad \forall t, \forall \omega \\
\delta_{t, \omega}=\delta_{t, \omega}^{+}-\delta_{t, \omega}^{-} \quad \forall t, \forall \omega \\
0 \leq \delta_{t, \omega}^{+} \leq W_{t, \omega} \quad \forall t, \forall \omega \\
0 \leq \delta_{t, \omega}^{-} \leq W^{\max } \quad \forall t, \forall \omega \\
\left(P_{t, \omega}^{D}-P_{t, \omega^{\prime}}^{D}\right)\left(\lambda_{t, \omega}^{D}-\lambda_{t, \omega^{\prime}}^{D}\right) \geq 0 \quad \forall t, \forall \omega, \forall \omega^{\prime} \\
P_{t, \omega}^{D}=P_{t, \omega^{\prime}}^{D} \quad \forall t, \forall \omega, \forall \omega^{\prime}: \lambda_{t, \omega}^{D}=\lambda_{t, \omega^{\prime}}^{D} \\
P_{t, \omega}^{I}=P_{t, \omega^{\prime}}^{I} \quad \forall t, \forall \omega, \forall \omega^{\prime}: \lambda_{t, \omega}^{D}=\lambda_{t, \omega^{\prime}}^{D} \\
-\gamma P_{t, \omega}^{D} \leq P_{t, \omega}^{I} \leq \gamma P_{t, \omega}^{D} \quad \forall t, \forall \omega .
\end{gathered}
$$

The objective function in (1) maximizes WPP profit. The first and second summation terms represent WPP profits in DA and intraday markets, respectively, whereas the $3^{\text {rd }}$ and $4^{\text {th }}$ terms are WPP profit and cost, respectively, in the balancing market. In (1), we have $R^{-} \geq 1$ and $R^{+} \leq 1$ implying that the generation deficiency of WPP has a higher penalty and its surplus generation is bought with a less price.

Constraint (2) confines the offer of WPP in the DA market to its rated power. The WPP scheduled power in (3) is comprised of its offers in the DA and intraday markets and is limited to WPP rated power by (4). The power deviation of WPP with respect to its scheduled power is given by (5). The positive and negative power deviation results in a profit and cost for WPP, respectively, in the objective function (1). In order to extract the positive and negative deviations, (6)-(8) are imposed. Note that only one of $\delta^{+}$and $\delta^{-}$can be nonzero in one individual time period: $\delta^{+}\left(\delta^{-}\right)=0$ if $\delta^{-}\left(\delta^{+}\right) \neq 0$. It is noted that the maximum value of $\delta^{+}$occurs when $P^{\text {sch }}$ is equal to zero; thus, the upper limit of $\delta^{+}$is set to $W$ in (7). Also, the maximum of $\delta^{-}$occurs when $P^{\text {sch }}$ is equal to $W^{\max }$; therefore, its upper limit is set to $W^{\max }$ in (8). The fact that the offer curve of WPP is not scenario dependent is constrained by (9)-(11). The intraday market is in fact developed to modify the DA offer by a given value. The portion of intraday offer with respect to the DA market is expressed by (12), where the coefficient $\gamma$ is decided by the market operator.

\section{B. The Uncoordinated Model of Aggregated DR}

The proposed model for DR is formulated as [3]:

$L_{t, \omega}^{D}, L_{t, \omega}^{I}, L_{t, \omega}^{s c h} \sum_{t=1}^{N_{T}} \sum_{\omega=1}^{N_{\omega}} \rho_{\omega}\left\{\begin{array}{l}\lambda_{t, \omega}^{D} L_{t, \omega}^{D}+\lambda_{t, \omega}^{I} L_{t, \omega}^{I}+\lambda^{*} L_{t, \omega}^{D}+\lambda^{*} L_{t, \omega}^{I} \\ +\frac{1}{2 \sigma D_{0, t}}\left(L_{t, \omega}^{s c h}\right)^{2}\end{array}\right\}$ 
subject to:

$$
\begin{array}{ll}
L_{t, \omega}^{s c h}=L_{t, \omega}^{D}+L_{t, \omega}^{I} & \forall t, \forall \omega \\
0 \leq L_{t, \omega}^{D} \leq \eta_{1} D_{0, t} & \forall t, \forall \omega \\
0 \leq L_{t, \omega}^{s c h} \leq \eta_{1} D_{0, t} & \forall t, \forall \omega \\
\sum_{t=1}^{N_{T}} L_{t, \omega}^{s c h} \leq \mu \sum_{t=1}^{N_{T}} D_{0, t} \quad \forall \omega .
\end{array}
$$

The objective function in (13) maximizes DR profit: the first two summation terms are the money paid to demands with the normal energy price rates in DA and intraday markets. In addition, demands are paid an incentive payment with a fixed rate as modeled by the third and fourth summation terms. The last term of the summation expresses DR profit considering elastic demand by using the exponential demand consumption versus price and Taylor series of DR benefit function [3]. The scheduled curtailable DR power is given in (14) as the sum of DR powers in the DA and intraday markets. The upper limit of DA and scheduled load curtailments are limited by (15) and (16), respectively, as a fraction of initial load. Constraint (17) limits the scheduled curtailable load energy (in DA and intraday markets) to its upper limit of $\mu \sum_{t=1}^{N_{T}} D_{0, t}$. In fact, the demand can offer the energy volume of $\mu \sum_{t=1}^{N_{T}} D_{0, t}$ as the total amount of daily curtailable load energy like a generation capacity to participate in the DA and intraday markets. The last summation term in (13) makes the optimization problem nonlinear. This term can be linearized using the conventional piecewise linearization method [19]. Thus, the linearized objective function of (13) is expressed as:

$$
L_{t, \omega}^{D}, L_{t, \omega}^{I}, L_{t, \omega}^{s c h} \sum_{t=1}^{N_{T}} \sum_{\omega=1}^{N_{\omega}} \rho_{\omega}\left\{\begin{array}{l}
\lambda_{t, \omega}^{D} L_{t, \omega}^{D}+\lambda_{t, \omega}^{I} L_{t, \omega}^{I}+\lambda^{*} L_{t, \omega}^{D} \\
+\lambda^{*} L_{t, \omega}^{I}+\frac{1}{2 \sigma D_{0, t}} \sum_{l=1}^{N_{L}} S_{l} L_{l, t, \omega}^{s c h} l
\end{array}\right\}
$$

subject to:

$$
\begin{gathered}
L_{t, \omega}^{s c h}=\sum_{l=1}^{N_{L}} L_{l, t, \omega}^{s c h \_l} \quad \forall t, \forall \omega \\
\text { Constraints (14)-(17). }
\end{gathered}
$$

\section{The Uncoordinated Model of ESS}

The proposed model for ESS is formulated as:

$$
P_{t, \omega}^{c h, D}, P_{t, \omega}^{d c h, D}, P_{t, \omega}^{c h, I}, P_{t, \omega}^{d c h, I} \sum_{t=1}^{N_{T}} \sum_{\omega=1}^{N_{\omega}} \rho_{\omega}\left\{\begin{array}{l}
\lambda_{t, \omega}^{D}\left(P_{t, \omega}^{d c h, D}-P_{t, \omega}^{c h, D}\right)+ \\
\lambda_{t, \omega}^{I}\left(P_{t, \omega}^{d c h, I}-P_{t, \omega}^{c h, I}\right)
\end{array}\right\}
$$

subject to:

$$
\begin{gathered}
P_{t, \omega}^{c h, s c h}=P_{t, \omega}^{c h, D}+P_{t, \omega}^{c h, I} \quad \forall t, \forall \omega \\
P_{t, \omega}^{d c h, s c h}=P_{t, \omega}^{d c h, D}+P_{t, \omega}^{d c h, I} \quad \forall t, \forall \omega \\
0 \leq P_{t, \omega}^{c h, D} \leq P^{\max } y_{t} \quad \forall t, \forall \omega \\
0 \leq P_{t, \omega}^{d c h, D} \leq P^{\max }\left(1-y_{t}\right) \quad \forall t, \forall \omega \\
0 \leq P_{t, \omega}^{c h, s c h} \leq P^{\max } y_{t} \quad \forall t, \forall \omega \\
0 \leq P_{t, \omega}^{d c h, s c h} \leq P^{\max }\left(1-y_{t}\right) \quad \forall t, \forall \omega \\
E_{t, \omega}^{D}=E_{0}+d u_{t}\left(\eta_{c h} P_{t, \omega}^{c h, D}-\frac{1}{\eta_{d c h}} P_{t, \omega}^{d c h, D}\right) \quad \forall t=1, \forall \omega
\end{gathered}
$$

$$
\begin{gathered}
E_{t, \omega}^{D}=E_{t-1, \omega}^{D}+d u_{t}\left(\eta_{c h} P_{t, \omega}^{c h, D}-\frac{1}{\eta_{d c h}} P_{t, \omega}^{d c h, D}\right) \quad \forall t \geq 2, \forall \omega \\
E_{t, \omega}^{s c h}=E_{0}+d u_{t}\left(\eta_{c h} P_{t, \omega}^{c h, s c h}-\frac{1}{\eta_{c h}} P_{t, \omega}^{d c h, s c h}\right) \quad \forall t=1, \forall \omega \\
E_{t, \omega}^{s c h}=E_{t-1, \omega}^{s c h}+d u_{t}\left(\eta_{c h} P_{t, \omega}^{c h, s c h}-\frac{1}{\eta_{c h}} P_{t, \omega}^{d c h, s c h}\right) \quad \forall t \geq 2, \forall \omega \\
E^{\min } \leq E_{t, \omega}^{D} \leq E^{\max } \quad \forall t, \forall \omega \\
E^{\min } \leq E_{t, \omega}^{s c h} \leq E^{\max } \quad \forall t, \forall \omega .
\end{gathered}
$$

The profit of ESS is maximized by the objective function of (20), where the first and second summation terms refer to ESS profit in the DA and intraday markets, respectively. The scheduled charging power of ESS is sum of its offers in DA and intraday markets as expressed by (21). Similarly, the discharging power is given by (22). The charging and discharging offer of ESS in the DA market is constrained by (23) and (24), respectively, where $y_{t}$ as a binary variable determines whether the ESS is being charged. Similarly, (25) and (26) constraint the scheduled charging and discharging offers of ESS, respectively. The stored energy of ESS in the DA market is formulated by (27) and (28) for the first and other time periods, respectively. In these equations, the charging and discharging efficiency of ESS is taken into account. Equations (29) and (30) similarly give the scheduled energy of ESS. Finally, (31) and (32) confine ESS energy to its lower and upper limits.

\section{The Proposed Model for VPP}

In the previous subsections, uncoordinated models of WPP, DR, and ESS are reviewed. As it can be seen in Fig. 1, in the uncoordinated operation, WPPs and ESSs submit their generation scheduling offer and the DRPs submit their reduction bid, independently. According to the proposed model in Fig. 1, for the coordinated scheme, for the joint operation of WPP, DR, and ESS, a central decision maker is required. The so called VPP is directly responsible for participating in all three markets (day-ahead, intraday, and balancing markets). Accordingly, firstly, VPP gathers the information of WPP (e.g., predicted wind power), ESS (status of charge, charging and discharging efficiencies) and DRP (e.g., load shifting/reduction capability, initial hourly load) and afterwards, decides the best offering strategy by forecasting market prices based on the latest information, technical constraints and market rules. Here, we formulate a VPP model consisting of a WPP, DR, and ESS. Using the coordinated operation of these resources, the profit of VPP in different markets are higher than uncoordinated operations. The complete form of the proposed VPP model is as follows:

$$
\begin{aligned}
& P_{t, \omega}^{D, V P P}, P_{t, \omega}^{I, V P P}, \delta_{t, \omega}^{+, V P P} \\
& \delta_{t, \omega}^{-, V P P}, L_{t, \omega}^{s c h, V P P}
\end{aligned} \sum_{t=1}^{N_{T}} \sum_{\omega=1}^{N_{\omega}} \rho_{\omega}\left\{\begin{array}{l}
\lambda_{t, \omega}^{D} P_{t, \omega}^{D, V P P}+\lambda_{t, \omega}^{I} P_{t, \omega}^{I, V P P} \\
+\lambda_{t, \omega}^{D} R_{t, \omega}^{+} \delta_{t, \omega}^{+, V P P} \\
-\lambda_{t, \omega}^{D} R_{t, \omega}^{-} \delta_{t, \omega}^{-, V P P} \\
+\frac{1}{2 \sigma D_{0, t}} \sum_{l=1}^{N_{L}} S_{l} L_{l, t, \omega}^{s c h l l, V P P}
\end{array}\right\}
$$


subject to:

$$
\begin{aligned}
& 0 \leq P_{t, \omega}^{D, V P P} \leq W^{\max }+\eta_{1} D_{0, t}+P^{\max }\left(1-y_{t}\right)-P^{\max } y_{t} \\
& \forall t, \forall \omega \\
& P_{t, \omega}^{s c h, V P P}=P_{t, \omega}^{D, V P P}+P_{t, \omega}^{I, V P P} \quad \forall t, \forall \omega \\
& 0 \leq P_{t, \omega}^{s c h, V P P} \leq W^{\max }+\eta_{1} D_{0, t}+P^{\max }\left(1-y_{t}\right)-P^{\max } y_{t} \\
& \forall t, \forall \omega \\
& \delta_{t, \omega}=W_{t, \omega}+L_{t, \omega}^{s c h, V P P}-P_{t, \omega}^{s c h, V P P} \quad \forall t, \forall \omega \\
& \delta_{t, \omega}=\delta_{t, \omega}^{+, V P P}-\delta_{t, \omega}^{-, V P P} \quad \forall t, \forall \omega \\
& 0 \leq \delta_{t, \omega}^{+, V P P} \leq W_{t, \omega}+L_{t, \omega}^{s c h, V P P} \quad \forall t, \forall \omega \\
& 0 \leq \delta_{t, \omega}^{-, V P P} \leq W^{\max }+\eta_{1} D_{0, t}+P^{\max }\left(1-y_{t}\right)-P^{\max } y_{t} \\
& \forall t, \forall \omega \\
& \left(P_{t, \omega}^{D, V P P}-P_{t, \omega^{\prime}}^{D, V P P}\right)\left(\lambda_{t, \omega}^{D}-\lambda_{t, \omega^{\prime}}^{D}\right) \geq 0 \quad \forall t, \forall \omega, \forall \omega^{\prime} \\
& P_{t, \omega}^{D, V P P}=P_{t, \omega^{\prime}}^{D, V P P} \quad \forall t, \forall \omega, \forall \omega^{\prime}: \lambda_{t, \omega}^{D}=\lambda_{t, \omega^{\prime}}^{D} \\
& P_{t, \omega}^{I, V P P}=P_{t, \omega^{\prime}}^{I, V P P} \quad \forall t, \forall \omega, \forall \omega^{\prime}: \lambda_{t, \omega}^{D}=\lambda_{t, \omega^{\prime}}^{D} \\
& -\gamma P_{t, \omega}^{D, V P P} \leq P_{t, \omega}^{I, V P P} \leq \gamma P_{t, \omega}^{D, V P P} \quad \forall t, \forall \omega \\
& L_{t, \omega}^{s c h, V P P}=L_{t, \omega}^{D, V P P}+L_{t, \omega}^{I, V P P} \quad \forall t, \forall \omega \\
& 0 \leq L_{t, \omega}^{D, V P P} \leq \eta_{1} D_{0, t} \quad \forall t, \forall \omega \\
& 0 \leq L_{t, \omega}^{s c h, V P P} \leq \eta_{1} D_{0, t} \quad \forall t, \forall \omega \\
& \sum_{t=1}^{N_{T}} L_{t, \omega}^{s c h, V P P} \leq \mu \sum_{t=1}^{N_{T}} D_{0, t} \quad \forall \omega \\
& L_{t, \omega}^{s c h, V P P}=\sum_{l=1}^{N_{L}} L_{l, t, \omega}^{s c h l, V P P} \quad \forall t, \forall \omega \\
& L_{t, \omega}^{I, V P P}=L_{t, \omega^{\prime}}^{I, V P P} \quad \forall t, \forall \omega, \forall \omega^{\prime}: \lambda_{t, \omega}^{D}=\lambda_{t, \omega^{\prime}}^{D} \\
& P_{t, \omega}^{s c h, V P P}=P_{t, \omega^{\prime}}^{s c h, V P P} \quad \forall t, \forall \omega, \forall \omega^{\prime}: \lambda_{t, \omega}^{D}=\lambda_{t, \omega^{\prime}}^{D} \\
& E_{t, \omega}^{D, V P P}=E_{0}+d u_{t}\left(\eta_{c h} P_{t, \omega}^{c h, D, V P P}-\frac{1}{\eta_{d c h}} P_{t, \omega}^{d c h, D, V P P}\right) \\
& \forall t=1, \forall \omega \\
& E_{t, \omega}^{D, V P P}=E_{t-1, \omega}^{D, V P P}+d u_{t}\left(\eta_{c h} P_{t, \omega}^{c h, D, V P P}-\frac{1}{\eta_{d c h}} P_{t, \omega}^{d c h, D, V P P}\right) \\
& \forall t \geq 2, \forall \omega \\
& E^{\min } \leq E_{t, \omega}^{D, V P P} \leq E^{\max } \quad \forall t, \forall \omega \\
& 0 \leq P_{t, \omega}^{c h, D, V P P} \leq P^{\max } y_{t} \quad \forall t, \forall \omega \\
& 0 \leq P_{t, \omega}^{d c h, D, V P P} \leq P^{\max }\left(1-y_{t}\right) \quad \forall t, \forall \omega \\
& E_{t, \omega}^{s c h, V P P}=E_{0}+d u_{t}\left(\eta_{c h} P_{t, \omega}^{c h, s c h, V P P}-\frac{1}{\eta_{d c h}} P_{t, \omega}^{d c h, s c h, V P P}\right) \\
& \forall t=1, \forall \omega \\
& E_{t, \omega}^{s c h, V P P}=E_{t-1, \omega}^{s c h, V P P}+d u_{t}\left(\eta_{c h} P_{t, \omega}^{c h, s c h, V P P}-\frac{1}{\eta_{d c h}} P_{t, \omega}^{d c h, s c h, V P P}\right) \\
& \forall t \geq 2, \forall \omega \\
& E^{\min } \leq E_{t, \omega}^{s c h, P P P} \leq E^{\max } \quad \forall t, \forall \omega \\
& 0 \leq P_{t, \omega}^{c h, s c h, V P P} \leq P^{\max } y_{t} \quad \forall t, \forall \omega \\
& 0 \leq P_{t, \omega}^{d c h, s c h, V P P} \leq P^{\max }\left(1-y_{t}\right) \quad \forall t, \forall \omega .
\end{aligned}
$$

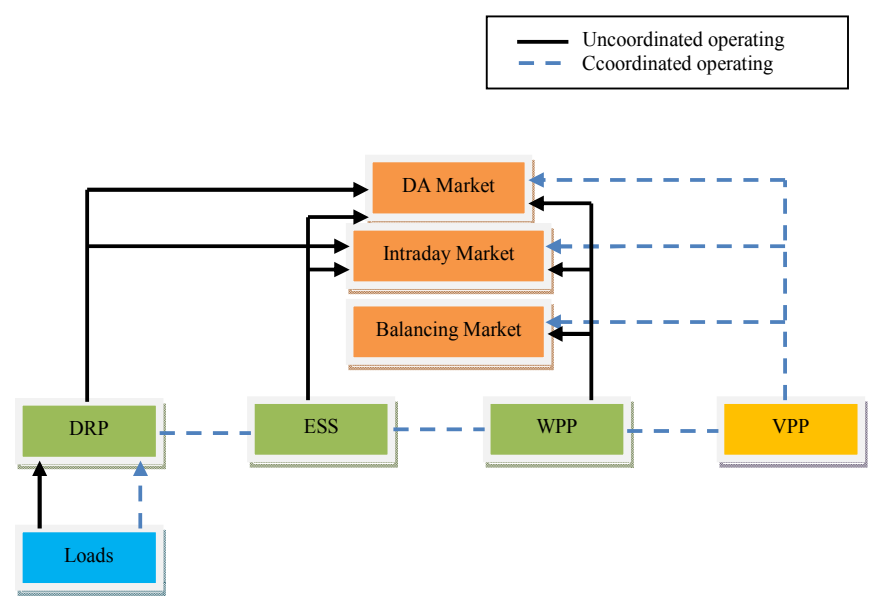

Fig. 1. Schematic representation of the proposed coordinated configuration.

The profit of VPP is maximized by objective function of (33), where the first two summation terms are VPP profit in DA and intraday markets, the third and fourth terms represent VPP income and cost due to power positive and negative imbalances in the balancing market, and the last summation term is the VPP income due to curtailable demands. The offer capacity of VPP is constrained by (34) that includes three parts of $W^{\max }$ as the WPP generation capacity, $\eta_{1} D_{0, t}$ as the DR generation capacity, and $P^{\max }\left(1-y_{t}\right)-P^{\max } y_{t}$ as the ESS generation capacity. The scheduled power of VPP in (35) is sum of its offers in DA and intraday markets. The VPP scheduled offer is constrained in (36) similar to (35). The offer deviation from the scheduled value is given by (37). Constraints (38)-(40) extract the positive and negative offer deviation as done in (6)-(8). The scenario characteristic of the offer curve is modeled by (41)-(43) like as done in (9)-(11). The offer adjustment limits are set by (44). The scheduled curtailable load of VPP is sum of its offers in the DA and intraday markets as formulated by (45). Equations (46)-(47) confines DA and scheduled curtailable load amounts to their limits. Constraint (48) limits the total amount of curtailable load energy of VPP to a preset percentage of initial load energy. Equation (49) calculates the scheduled curtailable load offer from linearized segments. Equations (50)-(51) imposes non-anticipative condition for the intraday and scheduled energy offer of VPP, respectively. The energy level of ESS of VPP in the DA market is calculated by (52)-(54) and its charging and discharging offer is constrained by (55)-(56). Similarly, equations (57)-(61) set constrains for the scheduled ESS offer of VPP.

It is noted that in the proposed model, the VPP is like to a package that includes WPP, DR and ESS. Therefore, the VPP has a variable power generation such as $P^{s c h,} V P P$, where it includes WPP generated power, DR shifted/reduced power, and ESS charging/discharging power. Hence, it is limited to $W^{\max }, \eta_{l} D_{0, t}, P^{\max }\left(1-y_{t}\right)$ and $-P^{\max } y_{t}$ due to WPP generated power, DR shifted/reduced power, and ESS charging/discharging power, respectively. Also, the WPP, DR and ESS constraints should be included. Hence, equations (37)-(43) are used in the proposed model to satisfy WPP requirements. Also, DR part of VPP is modeled as (44)-(51) by defining the variable $L^{\text {sch, } V P P}$ for the VPP. 
Note, this variable shows the load shifting/reduction in VPP. Finally, the constraints (52)-(61) formulate the ESS part of the VPP using variables $P_{t, \omega}^{c h, s c h, V P P}$ and $P_{t, \omega}^{c h, s c h, V P P}$.

\section{E. Stochastic Programming Method}

WPPs face two major sources of uncertainty: availability of the wind generation and market prices (DA, ID and Balancing). In addition, DRP and ESS problem described above is subject to the uncertainty of DA and ID market prices. In order to deal with these uncertainties, the coordinated offering strategy of VPP has been modeled as stochastic processes. To this end, a multi-stage stochastic programming is employed to solve offering strategy of VPP. Each stage refers to each market (DA, ID and Balancing) including first-stage (here-and-now), second-stage (wait-andsee 1 ) and third-stage (wait-and-see2). Decision making of the first stage should be specified before the realization of the scenarios. Accordingly, the first-stage decision variables are related to the DA market variables. When the DA market prices are known for each time horizon, the decision variables of the second stage should be determined for each possible realization of DA market prices. Finally, decision variables of the third stage of the stochastic programming refers to the balancing market. In this paper, we follow a stochastic programming method based on [20-22].

In the proposed method, we assume that WPP generation as well as prices of the DA, intraday, and balancing markets are uncertain parameters. These parameters are forecasted in advance and we formulate their forecast errors using appropriate Probability Distribution Functions (PDFs) [20]. Then, the roulette wheel mechanism [21] is used to generate possible joint scenarios. In order to enhance the computational efficiency of the stochastic programming, the Kantorovich method [22] is applied for scenario reduction. The number of scenarios that are generated for the above-mentioned stochastic parameters in the proposed method are as:

- $\quad N_{W}$ scenarios for the wind power generation.

- $N_{D}$ scenarios for DA market price $\left(\lambda^{D}\right)$.

- $\quad N_{I}$ scenarios for intraday market price $\left(\lambda^{I}\right)$.

- $\quad N_{R}$ scenarios for balancing market price $\left(R^{+}, R^{-}\right)$.

As a result, the total number of combinational scenarios in the proposed method will be $N_{W} \cdot N_{D} \cdot N_{I} \cdot N_{R}$. In the case study, we assumed $10,10,5,6$ for $N_{W}, N_{D}, N_{I}$ and $N_{R}$, respectively, resulting in total number of 3000 combinational scenarios. Finally, noted that further details about the proposed scenario generation\reduction algorithm can be found in [23].

\section{NUMERICAL RESULTS}

The proposed method is here tested on the Spanish Sotavento wind farm [24] with the rated capacity of 17.56 MW. This WPP is considered with an ESS with the specifications listed in Table II [25]. The system load data and energy prices for DA and intraday markets are adopted from the Iberian Peninsula market [26]. Our study is carried out on a week spanning 7-13 March 2010 of this market.
In the following subsections of A-D, we focus on the first day of the week (March 7 of 2010) to better focus on the results. However, in subsection E, we present results for the whole week. Parameters of the proposed method as used in the simulations are $0.3,0.04,-0.3,0.2$, and $0.3 \$ / \mathrm{MWh}$ for $\gamma, \mu, \sigma$, $\eta_{1}$, and $\lambda^{*}$, respectively [23]. The DA market price is a random parameter in the proposed method; its mean values are depicted in Fig. 2 for days of the week under study [26].

In Fig. 3, the initial hourly load before curtailing is presented for days of the week [26]. In order to solve optimization problems, we used here the GAMS software and CPLEX solver [27]. In the subsequent parts, results are presented in different uncoordinated and coordinated modes in order to compare them and evaluate the performance of the proposed joint operation of VPP. Results presented in following subsections are resulted from stochastic programming as a weighted sum of parameters using probability of scenarios. It is noted that, the day-ahead scheduling power for WPP, ESS and DR are defined by variables $\quad P_{t, \omega}^{D, V P P}, L_{t, \omega}^{D, V P P}$ and $\quad\left\{P_{t, \omega}^{c h, D, V P P}, P_{t, \omega}^{d c h, D, V P P}\right\}, \quad$ with superscript $D$, respectively. The hourly power scheduling of WPP, ESS, DR and VPP is obtained using objective function (33), equations (45) and (52)-(53).

\section{A. Optimal Uncoordinated Operation of WPP}

The problem in this section is formulated by (1)-(12) as a Linear Programming (LP) model. The optimal hourly bid of WPP in the DA and intraday markets as obtained after solving the model is plotted in Fig. 4. As seen, the WPP participated with its full capacity (17.56 MW) when the price is higher (see Fig. 2) in order to maximize its profit in DA, intraday, and balancing markets. The hourly expected profit of WPP is also depicted in Fig. 5. As seen, WPP obtain its profit majorly from the DA market. Total profit of WPP from the three markets is equal to $\$ 4721$.

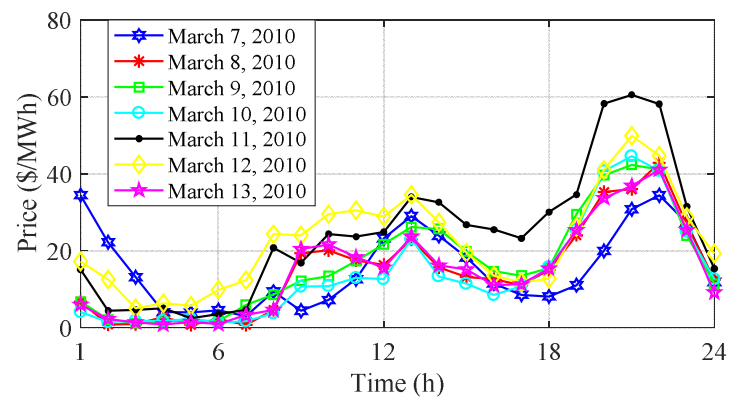

Fig. 2. Mean energy price of the day-head market in March 7-13 of 2010 [26]

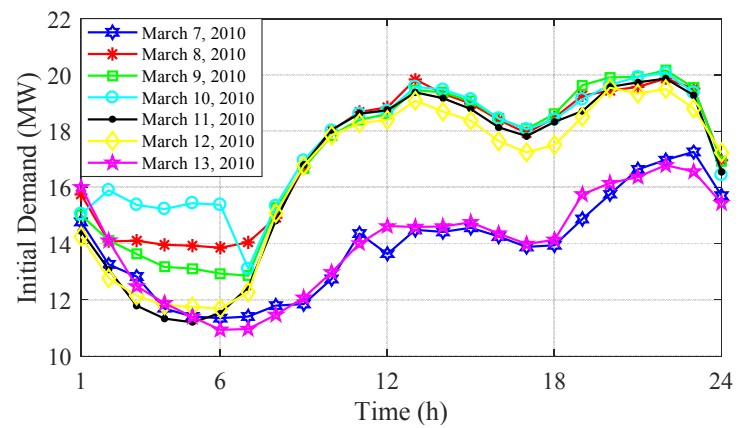

Fig. 3. Initial load demand in March 7-13 of 2010 [26] 
TABLE II. ENERGY STORAGE SYSTEM SPECIFICATIONS

\begin{tabular}{|c|c|}
\hline Quantity & Value \\
\hline Initial energy $(\mathrm{MWh})$ & 5 \\
\hline Minimum energy $(\mathrm{MWh})$ & 2 \\
\hline Maximum energy $(\mathrm{MWh})$ & 25 \\
\hline Maximum charging/discharging power $(\mathrm{MW})$ & 4 \\
\hline
\end{tabular}

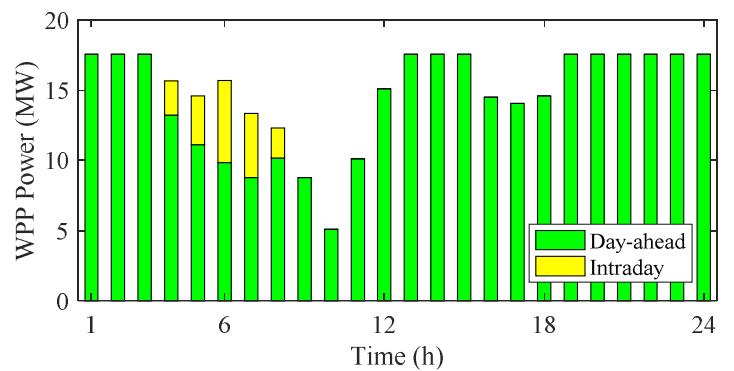

Fig. 4. Optimal bid of WPP in March 7 of 2010

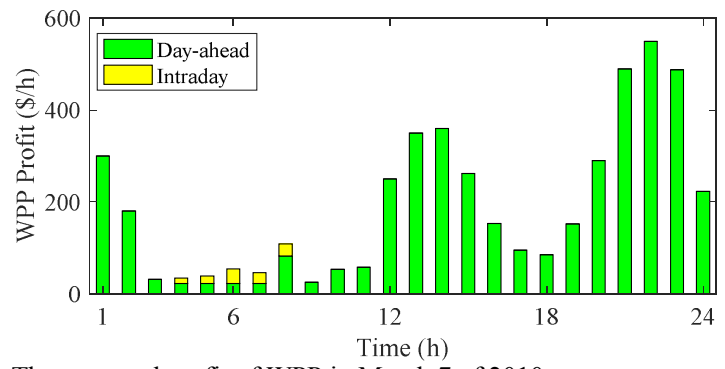

Fig. 5. The expected profit of WPP in March 7 of 2010

\section{B. Optimal Uncoordinated Operation of Aggregated DR}

The problem in this case is formulated by (14)-(19) as an LP model. The optimal curtailable DR offer and its profit is depicted in Fig. 6 and Fig. 7, respectively, for March 7 of 2010. As seen, DR is more sensitive than WPP to price signals and its offer happens only in peak hours of the market. That is, only when the energy price is high, it is profitable for DR to participate in the market.

As seen in Fig. 3 (the March 7, 2010 curve), the load demand at peak hours is about $17 \mathrm{MW}$ before DR. The participation of DR reduces this peak demand by about $3 \mathrm{MW}$ at peak hours as seen in Fig. 6. Total profit of DR from the three markets of DA, intraday, and balancing is equal to $\$ 1200$.

\section{Optimal Uncoordinated Operation of ESS}

The optimization problem in this case is modeled by (20)(32) as an MILP. The optimal offer of ESS in the DA market is plotted in Fig. 8 and the expected profit of ESS is shown in Fig. 9.

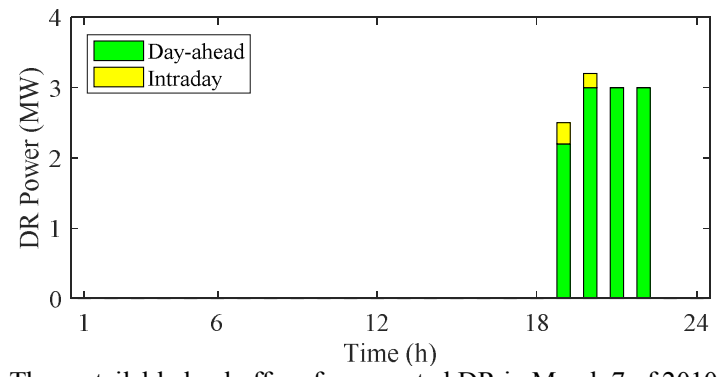

Fig. 6. The curtailable load offer of aggregated DR in March 7 of 2010

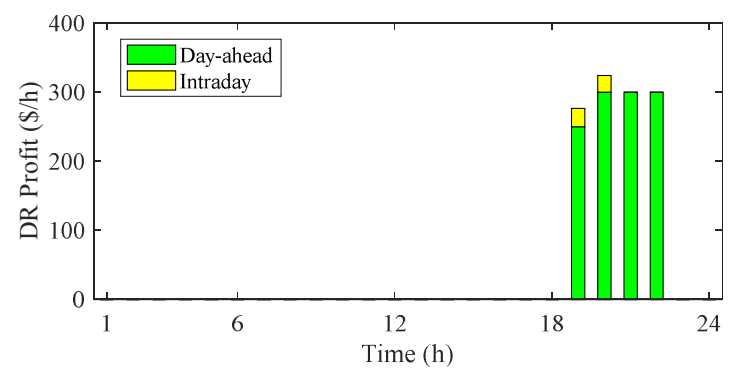

Fig. 7. The expected profit of aggregated DR in March 7 of 2010

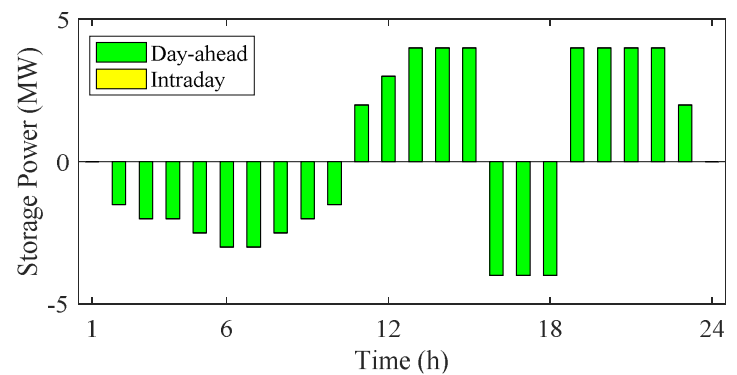

Fig. 8. The optimal offer of ESS in March 7 of 2010

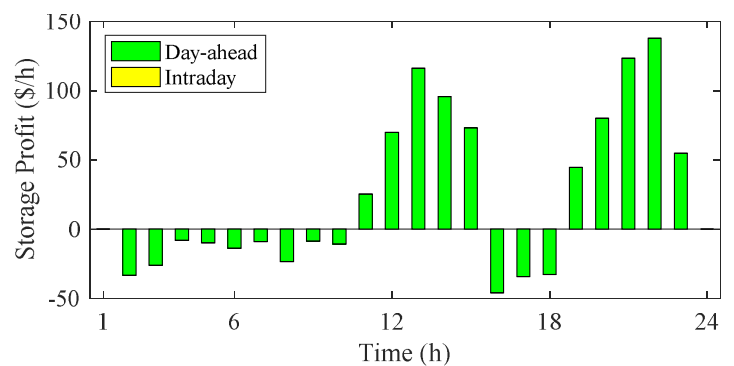

Fig. 9. The expected profit of ESS in March 7 of 2010

As seen in Fig. 8, the ESS is charged in off-peak and midpeak hours and it is discharged in on-peak hours. That is, the energy is bought in low-tariff hours and it is sold in high-tariff hours in order to maximize ESS profit.

Although the ESS optimization problem of (20)-(32) includes its offers in both DA and intraday markets, it is not profitable for ESS to participate in the intraday market and then, it participates only in the DA market as seen in Fig. 8. Total profit of ESS is equal to $\$ 662$.

\section{Optimal Coordinated Operation of VPP}

The VPP in this case includes the joint optimization of WPP, DR, and ESS as formulated by (33)-(61) as an MILP model. The VPP optimal offer and its expected profit are plotted in Fig. 10 and Fig. 11, respectively.

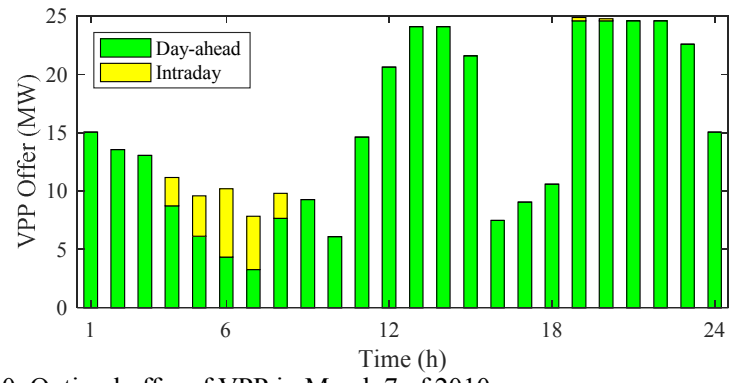

Fig. 10. Optimal offer of VPP in March 7 of 2010 


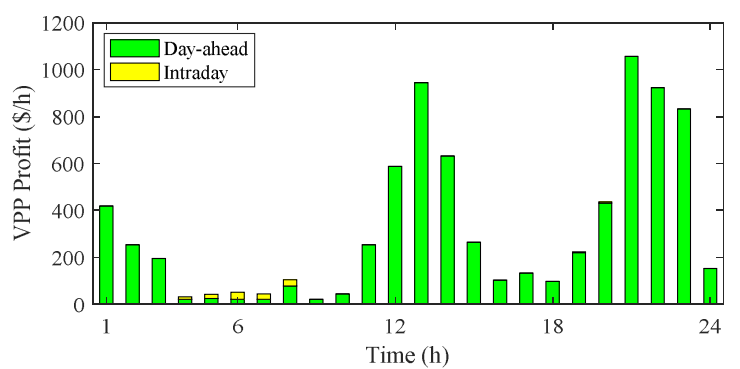

Fig. 11. The expected profit of VPP in March 7 of 2010

As seen in Fig. 10, the VPP offer has increased to about 25 MW at peak hours compared with the uncoordinated operation of WPP with 17.56 MW offer. In fact, VPP employs DR and ESS in addition to WPP as generation assets to more increase its profit at peak hours with higher tariffs. Total expected profit of VPP over the 24 hours has been $\$ 7810$. In the subsection, we compare the profit of VPP with individual uncoordinated operations of WPP, DR, and ESS.

\section{E. Comparison}

Results of uncoordinated operations of WPP, DR, and ESS as well as the coordinated operation of VPP are presented in previous subsections for the first day (7 March, 2010) of the week under study. In Table III, the summary of expected profits of uncoordinated and coordinated cases are presented for all days of the week under study. Also, sum of profits of the three uncoordinated operations is shown in the table. In addition, the coordinated operation of VPP (including WPP, DR, and ESS) is reported in the second part of Table III. Under column "7" of this table, profits of $\$ 4721$ (for WPP), $\$ 1200$ (for DR), \$662 (for ESS), and \$7810 (for VPP) are previously reported in preceding subsections. Considering the first day of the week (March 7 of 2010) in this table, sum of uncoordinated profits of WPP, DR, and ESS is equal to $\$ 6583$ $(4721+1200+662)$, whereas their coordinated operations in the VPP results in the profit of $\$ 7810$, which is considerably higher than uncoordinated operations (18.64\%). The gain in profit due to the coordinated VPP with respect to sum of uncoordinated operations is reported in the last row of the table for all days of the week. A higher profit implies a higher incentive for VPP to be participated in electricity markets using our proposed model. In other words, the proposed model increases the ability of WPPs in electricity markets without any need to supportive or subsidizing mechanisms.

This trend finally rises the penetration level of renewable energies in electricity markets. Not only the WPP power increases under the VPP framework, but also DR and ESS participate more effectively. For instance, the participation of DR in the DA market for the two cases is plotted in Fig. 112 for 7 March 2010. As seen, in the uncoordinated operation, the DR participates in limited hours with only positive values implying only load curtailment. However, in case of VPP coordinated operation, DR participates in extended hours with both positive and negative offers. The ultimate result is that the expected profit of the VPP increases in case of coordinated operation. In addition to increasing the profit of VPP, total energy supplied by WPP, ESS, and DR in the three markets also increase. In Table IV, the energy supplied by coordinated and uncoordinated cases is mentioned for the considered week.
TABLE III. EXPECTED PROFIT OF UNCOORDINATED AND COORDINATED CASES IN MARCH 7-13 OF 2010

\begin{tabular}{|c|c|c|c|c|c|c|c|c|}
\hline \multicolumn{2}{|c|}{ Day of week } & 7 & 8 & 9 & 10 & 11 & 12 & 13 \\
\hline \multirow{4}{*}{$\begin{array}{c}\text { Uncoordinated } \\
\text { operation }\end{array}$} & WPP & 4721 & 6524 & 9643 & 5235 & 10451 & 8345 & 7340 \\
\hline & DR & 1200 & 1520 & 1800 & 1621 & 2111 & 1851 & 1901 \\
\hline & ESS & 662 & 650 & 980 & 730 & 1112 & 1051 & 1023 \\
\hline & Sum & 6583 & 8694 & 12423 & 7586 & 13674 & 11247 & 10264 \\
\hline \multirow{5}{*}{$\begin{array}{c}\text { Coordinated } \\
\text { operation (VPP) }\end{array}$} & WPP & 4728 & 6534 & 9648 & 5241 & 10463 & 8353 & 7347 \\
\hline & DR & 2414 & 2602 & 2578 & 2453 & 3843 & 2913 & 2949 \\
\hline & ESS & 668 & 656 & 985 & 737 & 1117 & 1055 & 1029 \\
\hline & Sum & 7810 & 9792 & 13211 & 8431 & 15423 & 12321 & 11325 \\
\hline & Gain (\%) & 18.64 & 12.63 & \begin{tabular}{|l|l|}
6.34 \\
\end{tabular} & 11.14 & 12.79 & 9.55 & 10.34 \\
\hline
\end{tabular}

TABLE IV. ENERGY SUPPLIED BY WPP, ESS, AND DR IN 7-13 OF MARCH 2010 IN UNCOORDINATED AND COORDINATED CASES

\begin{tabular}{|c|c|c|c|c|c|c|c|c|}
\hline \multicolumn{2}{|c|}{ Day of week } & 7 & 8 & 9 & 10 & 11 & 12 & 13 \\
\hline \multirow{4}{*}{$\begin{array}{c}\text { Energy supplied } \\
\text { by uncoordinated } \\
\text { operations } \\
\text { (MWh) }\end{array}$} & WPP & 4721 & 6524 & 9643 & 5235 & 10451 & 8345 & 7340 \\
\hline & DR & 1200 & 1520 & 1800 & 1621 & 2111 & 1851 & 1901 \\
\hline & ESS & 662 & 650 & 980 & 730 & 1112 & 1051 & 1023 \\
\hline & Sum & 6583 & 8694 & 12423 & 7586 & 13674 & 11247 & 10264 \\
\hline \multirow{4}{*}{$\begin{array}{c}\text { Energy supplied } \\
\text { by coordinated } \\
\text { operation of VPP } \\
\text { (MWh) }\end{array}$} & WPP & 4728 & 6534 & 9648 & 5241 & 10463 & 8353 & 7347 \\
\hline & DR & 2414 & 2602 & 2578 & 2453 & 3843 & 2913 & 2949 \\
\hline & ESS & 668 & 656 & 985 & 737 & 1117 & 1055 & 1029 \\
\hline & Sum & 7810 & 9792 & 13211 & 8431 & 15423 & 12321 & 11325 \\
\hline
\end{tabular}

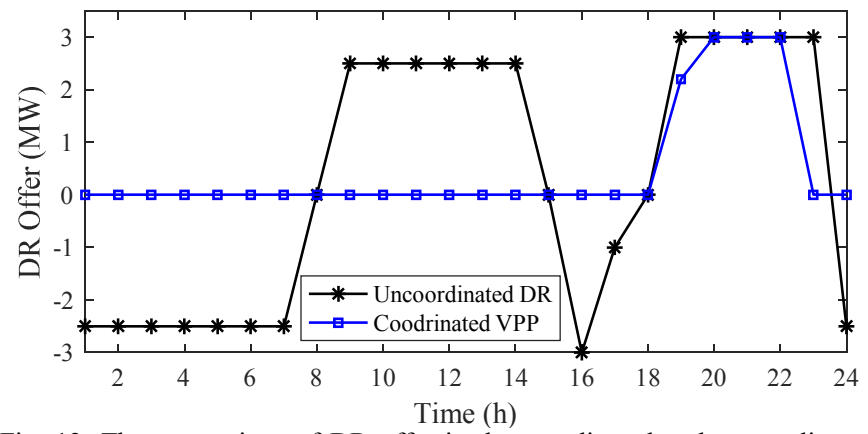

Fig. 12. The comparison of DR offer in the coordinated and uncoordinated operations in the day-head market in 7 March 2010

For instance, in the first day, total energy supplied by uncoordinated operations of WPP, DR, and ESS is 6583 $\mathrm{MWh}$, whereas it is $7810 \mathrm{MWh}$ in case of coordinated operation of VPP. Considering the whole week, total supplied energy over the week is $70.471 \mathrm{GWh}$ and $78.313 \mathrm{GWh}$ for uncoordinated and coordinated operations, respectively. This means that penetration level of renewable energies (WPP+ESS+DR) increases by $11.1 \%$ by the coordinated operation. As a result, the proposed coordinated operation increases not only the participation of renewables but also their profits, Table V. It is noted that these gains are obtained by a market-based framework, not by a subsidizing mechanism to support renewables. In other words, the main advantage of the proposed VPP framework is to promote renewable energy in electricity markets without any need to subside mechanisms, a development which is more consistent with electricity markets principles.

\section{F. Sensitivity Analysis for Degree of Coordination}

Here, the sensitivity analysis is done to determine the degree of coordination of the VPP's expected profit with respect to the variations of the capacity for WPP, DR and ESS as well as DA and ID market prices' change. 
Accordingly, the results have been shown in Table V. As it is seen in this table, the increment value $(\zeta \%)$ of each parameter has been changed from $0 \%$ to $30 \%$. Results show increasing trend in the value of expected profit for the VPP by increasing different parameters. In the last row of Table $\mathrm{V}$, the sensitivity factor of the expected profit of VPP with respect to the variations of each parameter has been addressed. For variations of the size for WPP, DR and ESS, the sensitivity factor has the approximate constant value. However, changing the DA and ID market prices reveals nonlinear change of the degree of coordination, because by changing the price, the degree of coordination of the WPP, DR and ESS will be changed for each hour.

TABle V. The Profit of VPP IN the Sensitive Analyze at 7 March 2010

\begin{tabular}{|c|c|c|c|c|c|c|}
\hline \multicolumn{2}{|c|}{ Increment (广\%) } & $\mathbf{0}$ & $\mathbf{1 0}$ & $\mathbf{2 0}$ & $\mathbf{3 0}$ & Deviation \\
\hline \multirow{4}{*}{$\begin{array}{c}\text { VPP profit } \\
\text { (\$) }\end{array}$} & WPP size & 7810 & 8283 & 8756 & 9228 & $+0.605^{*} \zeta$ \\
\cline { 2 - 7 } & DR size & 7810 & 8051 & 8293 & 8534 & $+0.308^{*} \zeta$ \\
\cline { 2 - 7 } & ESS size & 7810 & 7877 & 7944 & 8010 & $+0.086^{*} \zeta$ \\
\cline { 2 - 7 } & DA market price & 7810 & 8531 & 9372 & 10153 & $+(0.923$ to 1$) * \zeta$ \\
\cline { 2 - 7 } & ID market price & 7810 & 7943 & 8201 & 8653 & $+(0.17 \text { to } 0.36)^{*} \zeta$ \\
\hline
\end{tabular}

\section{CONCLUSIONS}

This paper presented a framework to maximize the profit of VPP, including a WPP, DR, and ESS in day-ahead, intraday, and balancing electricity markets. First, uncoordinated operations of WPP, DR, and ESS were formulated. Then, a model was proposed for the coordinated operation of VPP to maximize its profit for more efficiently managing power system assets. Scenarios of stochastic programming were generated using a joint scenario tree and the Kantorovich method was used for scenario reduction. The proposed framework was tested on a Spanish wind farm and was compared with previous methods. According to the obtained results, it was determined that the proposed VPP leads to both a higher participation of wind energy and a higher profit; for instance, in the first day of the week under study, it leads to $18.64 \%$ more profit and $11.1 \%$ more renewable energy participation compared with uncoordinated operations. The proposed method considers the simultaneous participation in three markets, so, a further work is needed to establish the optimization of scheduling in the intraday market. In fact, transactions in the intraday market are always in the form of bilateral contract which means that there could be lots of different prices in the same hour. Hence, investigation and experimentation into optimization in this market is strongly recommended.

\section{REFERENCES}

[1] J. M. Morales, A. J. Conejo, H. Madsen, P. Pinson, and M. Zugno, Integrating Renewables in Electricity Markets: Operational Problems. Springer US, 2014.

[2] L. Baringo and A. J. Conejo, "Offering Strategy of Wind-Power Producer: A Multi-Stage Risk-Constrained Approach," IEEE Trans. Power Syst., vol. 31, no. 2, pp. 1420-1429, 2016.

[3] E. Heydarian-Forushani, M. P. Moghaddam, M. K. Sheikh-El-Eslami, M. Shafie-khah, and J. P. S. Catalão, "Risk-Constrained Offering Strategy of Wind Power Producers Considering Intraday Demand Response Exchange," IEEE Trans. Sustain. Energy, vol. 5, no. 4, pp. 1036-1047, 2014.

[4] Á. J. Duque, E. D. Castronuovo, I. Sánchez, and J. Usaola, "Optimal operation of a pumped-storage hydro plant that compensates the imbalances of a wind power producer," Electr Pow Syst Res, vol. 81, no. 9, pp. 1767-1777, 2011.
[5] L. Baringo and A. J. Conejo, "Strategic Offering for a Wind Power Producer," IEEE Trans. Power Syst., vol. 28, pp. 4645-4654, 2013.

[6] M. Rahimiyan and L. Baringo, "Strategic Bidding for a Virtual Power Plant in the Day-Ahead and Real-Time Markets: A Price-Taker Robust Optimization Approach," IEEE Trans. Power Syst., vol. 31, no. 4, pp. $2676-2687,2016$

[7] H. Akhavan-Hejazi and H. Mohsenian-Rad, "Optimal Operation of Independent Storage Systems in Energy and Reserve Markets With High Wind Penetration," IEEE Trans. Smart Grid, vol. 5, no. 2, pp. 10881097, 2014.

[8] H. Mohsenian-Rad, "Optimal Bidding, Scheduling, and Deployment of Battery Systems in California Day-Ahead Energy Market," IEEE Trans. Power Syst., vol. 31, no. 1, pp. 442-453, 2016.

[9] H. Wu, M. Shahidehpour, A. Alabdulwahab, and A. Abusorrah, "Demand Response Exchange in the Stochastic Day-Ahead Scheduling With Variable Renewable Generation," IEEE Trans. Sustain. Energy, vol. 6, no. 2, pp. 516-525, 2015.

[10] H. T. Nguyen and L. B. Le, "Sharing Profit From Joint Offering of a Group of Wind Power Producers in Day Ahead Markets," IEEE Transactions on Sustainable Energy, vol. 9, pp. 1921-1934, Oct. 2018.

[11] C. Du, X. Wang, X. Wang, C. Shao and Y. Xiao, "A mechanism of intraday market design for promoting wind power integration," CSEE Journal of Power and Energy Systems, vol. 4, no. 3, pp. 293-298, September 2018.

[12] H. T. Nguyen, L. B. Le and Z. Wang, "A Bidding Strategy for Virtual Power Plants With the Intraday Demand Response Exchange Market Using the Stochastic Programming," IEEE Transactions on Industry Applications, vol. 54, no. 4, pp. 3044-3055, July-Aug. 2018.

[13] X. Fang, Q. Hu, F. Li, B. Wang and Y. Li, "Coupon-Based Demand Response Considering Wind Power Uncertainty: A Strategic Bidding Model for Load Serving Entities," IEEE Transactions on Power Systems, vol. 31, no. 2, pp. 1025-1037, March 2016.

[14] S. R. Konda, B. Mukkapati, L. K. Panwar, B. K. Panigrahi and R. Kumar, "Dynamic Energy Balancing Cost Model for Day Ahead Markets With Uncertain Wind Energy and Generation Contingency Under Demand Response," IEEE Transactions on Industry Applications, vol. 54, no. 5, pp. 4908-4916, Sept.-Oct. 2018

[15] J. Wu, B. Zhang and Y. Jiang, "Optimal day-ahead demand response contract for congestion management in the deregulated power market considering wind power," IET Generation, Transmission \& Distribution, vol. 12, no. 4, pp. 917-926, 2722018.

[16] H. Bitaraf and S. Rahman, "Reducing Curtailed Wind Energy Through Energy Storage and Demand Response," IEEE Transactions on Sustainable Energy, vol. 9, no. 1, pp. 228-236, Jan. 2018.

[17] J. Kazempour and B. F. Hobbs, "Value of Flexible Resources, Virtual Bidding, and Self-Scheduling in Two-Settlement Electricity Markets with Wind Generation-Part I: Principles and Competitive Model," IEEE Transactions on Power Systems, vol. 33, pp. 749-759, Jan. 2018.

[18] J. Kazempour and B. F. Hobbs, "Value of Flexible Resources, Virtual Bidding, and Self-Scheduling in Two-Settlement Electricity Markets With Wind Generation-Part II: ISO Models and Application," IEEE Transactions on Power Systems, vol. 33, no. 1, pp. 760-770, Jan. 2018.

[19] A. J. Wood and B. F. Wollenberg, Power Generation, Operation, and Control. Wiley, 2013.

[20] N. Amjady, F. Keynia, and H. Zareipour, "Wind Power Prediction by a New Forecast Engine Composed of Modified Hybrid Neural Network and Enhanced Particle Swarm Optimization," IEEE Trans. Sustain. Energy, vol. 2, no. 3, pp. 265-276, 2011.

[21] N. Amjady, J. Aghaei, and H. A. Shayanfar, "Stochastic Multiobjective Market Clearing of Joint Energy and Reserves Auctions Ensuring Power System Security," IEEE Trans. Power Syst., vol. 24, no. 4, pp. 18411854, 2009.

[22] N. Growe-Kuska, H. Heitsch, and W. Romisch, "Scenario reduction and scenario tree construction for power management problems," in 2003 IEEE Bologna Power Tech Conference Proceedings, pp. 7-12, 2003.

[23] J. Aghaei, M. Barani, M. Shafie-khah, A. A. Sánchez de la Nieta and J. P. S. Catalão, "Risk-Constrained Offering Strategy for Aggregated Hybrid Power Plant Including Wind Power Producer and Demand Response Provider," IEEE Trans. Sust. Energy, vol. 7, pp. 513-525, April 2016.

[24] Sotavento wind farm, Available online: http://www.sotaventogalicia.com. accessed 2017.

[25] E. Mashhour and S. Moghaddas-Tafreshi, "Mathematical modeling of electrochemical storage for incorporation in methods to optimize the operational planning of an interconnected micro grid," J Zhejiang Univ Sci C, vol. 11, no. 9, pp. 737-750, 2010.

[26] Market operator of the electricity market of the Iberian Peninsula, Available online: http://www.omel.es38T. accessed 2017.

[27] R. E. Rosenthal, General Algebraic Modeling System (GAMS) User Guide. Washington, DC, USA: GAMS Development Corporation, 2017. 
Ali Jamali is currently working toward the Ph.D. degree at the Shiraz University of Technology, Shiraz, Iran.

Jamshid Aghaei (M'12-SM'15) is a Professor at the Shiraz University of Technology, Shiraz, Iran. His research interests include renewable energy systems, smart grids, electricity markets, and power systems optimization.

Masoud Esmaili is an Assistance Professor at the Islamic Azad University, Tehran, Iran. His research interests include electricity markets, distributed generation, and distribution systems.

Ahmad Nikoobakht is an Assistance Professor at the Higher Education Center of Eghlid, Iran. His research interests include renewable energy systems, smart grids, electricity markets, and power systems operation.
Taher Niknam (M'14) is a Professor at the Shiraz University of Technology, Shiraz, Iran. His research interests include power system restructuring and impacts of distributed generations on power systems.

Miadreza Shafie-khah (M'13-SM'17) is an Assistant Professor at the University of Vaasa, Finland. His research interests include power market power system optimization, demand response and electric vehicles.

João P. S. Catalão (M'04-SM'12) is a Professor at the Faculty of Engineering of the University of Porto (FEUP), Porto, Portugal, and Research Coordinator at INESC TEC. His research interests include power system operations and planning, distributed renewable generation, demand response and smart grids. 